\title{
Electrical Impedance Changes in Many Sites of Brain in Paradoxical Sleep, Anesthesia, and Activity
}

\author{
JAMES B. RANCK, JR. ${ }^{1}$ \\ Department of Physiology, University of Michigan, Ann Arbor, Michigan 48104
}

Received January 26, 1970

\begin{abstract}
The electrical impedance of a part of brain approximately 1-1nm dimensions was measured with a four-electrode, very low current method in 61 male rats. The testing frequency was usually only $1000 \mathrm{~Hz}$, and only the magnitude of impedance was measured. Impedance increased in paradoxical sleep in 42 of the 61 sites and decreased at three sites in the pons. The greatest changes were in subiculum and presubiculum with changes usually more than $10 \%$ and up to $25-30 \%$. Intermediate changes of $2-10 \%$ were found in parasubiculum and entorhinal cortex. Most other changes were less than $4 \%$, and there is a suggestion of greater changes in the pretectal area. All sites with changes greater than $4 \%$ were within $1 \mathrm{~mm}$ of a pial or ependymal surface. During anesthesia with pentobarbital in 23 rats impedance increased in two, eight showed no change, and 13 decreased. During unrestrained spontaneous activity in a small familiar cage the impedance usually became either more variable or decreased generally to a maximum of $1-10 \%$, or both, but at a single site the response was not always the same. No tests beyond simple observation were used, and with this limited basis no clearer relation of impedance to behavior than simply to motor activity was apparent. In a change from quiet arousal to slow-wave sleep, or vice versa, there were no impedance changes. But at all sites activity ustrally had an effect. This was particularly marked in entorhinal cortex-parasubiculum and brain stem. Almost no other impedance changes were seen than of these three types. Impedance changes in brain are thus widespread and ozcur frequently in the usual behavior of rats. These results generally corroborate and expand the results of the Adey-Kado group with a different method with some advantages.
\end{abstract}

\section{Introduction}

Changes occur in the electrical impedance of parts of brain during normal functioning. The Adey-Kado group (2-5) have looked in detail at changes in the hippocampus, amygdala, and mesencephalic reticular formation of

1 This investigation was supported by Grant NB-04352 from The National Institutes of Health. The major part of the work, i.e., the preparation of electrodes, surgery, and running of the rats, was done by Mrs. Ann C. Maxwell. The histology was done by Miss Christine McBride. Misses Constance Jones and Barbara Dorencamp aided in some early stages of the investigation. 
cats during learning of a discriminative performance in a t-maze where changes of up to $15 \%$ are seen. The largest changes in normal functioning $(5-25 \%)$ have been seen in this laboratory in the subiculum and presubiculum (called simply "subicular area" in that report) of rats during paradoxical sleep (20). Impedance changes which are even larger are seen in many abnormal situations, but those changes in normal functioning are of especial significance.

This study has two major purposes. The first is to get a very general view of impedance changes by looking at spontaneous behavior of many rats and to look at many regions of brain. (This is in general a study of the forest, not the trees.) No single kind of behavior has been studied in detail, except for paradoxical sleep, which occurs in well defined episodes. The major changes the Adey-Kado group found were in a time-locked, highly trained behavior in a highly motivated state, so that the question of changes in less highly structured behavior more closely resembling the usual behavior of animals remains open. In general, changes occur in all 61 regions of brain tested and occur in paradoxical sleep and motor activity, so changes are widespread and common. The largest changes are in paradoxical sleep, and these are seen in almost all regions of brain.

The second major purpose of this study is methodological. The AdeyKado group measured impedance with a two-electrode method-the same electrodes pass current as record voltage, so that the poorly understood polarization impedance at the metal-to-liquid interface at the electrode surface (24) and the impedance of damaged tissue at the electrode tip contributed. A four-electrode method used in this study, in which two electrodes pass current and another two record voltage changes, effectively eliminates these two possible contaminants. While no direct comparisons are made with the Adey-Kado data, in general the results are consistent.

\section{Methods}

The method has been previously described in detail (20). Briefly, it utilizes four $75-\mu 92 \%$ platinum- $8 \%$ tungsten or $90 \%$ platinum- $10 \%$ iridium electrodes, insulated except for $75 \mu$ at the tip, and one large electrode at a distance. Current is passed between two of these electrodes, and voltages are recorded from two others. Sinusoidal currents of 1.5 to 10,000 $\mathrm{Hz}$ of less than $5 \times 10^{-8}$ amp are used, producing voltages of less than $50 \mu \mathrm{v}$. Filtering and averaging with a lock-in amplifier are used to record the impedance changes. Absolute values of phase angle of impedance are accurate to about $1^{\circ}$, and relative changes in magnitude of less than $1 \%$ can be measured. At $1000 \mathrm{~Hz}$, the measuring frequency most often used, the time constant of averaging was $1 \mathrm{sec}$. Spontaneous electrical activity can be recorded simultaneously from the voltage-recording electrodes since 
the impedance measuring signal is much less than the spontaneous electrical activity.

The small electrode tips were in a strainght line, all within $2.0 \mathrm{mmm}$, usually within $1.5 \mathrm{~mm}$ of each other. The small electrodes were all oriented in an anteroposterior direction, except for these in entorhinal cortex and parasubiculum, ventral subiculum, and 115 and 116 in the dorsal hippocampus, which were oriented in a mediolateral direction. Impedance can be recorded from two types of configurations [see Fig 1 of (20)]. (a) Current can be passed between one small electrode and the large one, so that current is flowing radially in the vicinity of the small current electrode. Let us call this a radial configuration. Voltage changes can then be recorded between any other two small electrodes. If the small electrode tips are arranged in an anteroposterior orientation and the small current electrode is the most anterior one, then we are effectively measuring impedance in a shell of tissue with its center at the small current electrode extending anterior to the electrode configuration and posterior to the most posterior voltage electrode. Let us call this an anterior configuration, and a posterior configuration is similarly defined. When the electrode tips are in a mediolateral configuration we will similarly define medial and lateral configuration. (b) Current can be passed between two small electrodes and voltage changes recorded between the other two small electrodes. This measures impedance in the vicinity of the two voltage-recording elctrode tips. Let us call this a linear configuration.

At the end of all measurements each rat was perfused with formalin, and frozen sections of the brain were cut and stained with cresyl violet for identification of electrode location. The impedance changes with pentobarbital anesthesia were measured after an intraperitoneal injection while the rat was being anesthetized before being perfused, so this change was recorded only once in each rat. Control measurements were made of the effect of lifting the rat out of the cage and of sticking him with a needle intraperitoneally.

Electrodes were put through stringent electrical testing before and after implantation, the details of which have been published (20). As each electrode was made it was given a number which became the number of the rat in which it was implanted. As can be seen from Table $1 \mathrm{~A}$ there were many electrodes or rats rejected, almost all because the electrodes did not meet electrical testing standards.

The magnitude of impedance at 1000 or $3200 \mathrm{~Hz}$ was measured in all rats. Measurements were made at other frequencies usually only when substantial changes in the magnitude at 1000 or $3200 \mathrm{~Hz}$ were seen. Phase angles of impedance were not measured. Therefore, for simplicity, the term "impedance" will be used to mean "magnitude of impedance." 
All rats were male Sprague-Dawley, weighing 250-500 g. Impedance electrodes and neocortical EEG electrodes (stainless steel screws) were implanted at least 3 weeks before recordings were made. Most of the rats were deprived of paradoxical sleep the night before recording sessions by the inverted flower-pot-in-water method. Often the rat was run for many days consecutively, in which case he was deprived for many consecutive nights but allowed to sleep during the day, so that it was under mild but never severe sleep deprivation. All observations were made while the rat was in a $29-\times 29-\times 29-\mathrm{cm}$ shielded cage of transparent plastic. Continuous recordings of impedance and spontaneous electrical activity from the same site or neocortical EEG were made over at least several hours while the rat was watched. The rat was usually observed and these observations recorded by a technician (A. C. M.), but other observations were made intermittently by myself. The rat was kept in this recording cage at other times also so that he was thoroughly familiar with it. Food and water were usually present.

\section{Results}

The only correlations with impedance seen were in paradoxical sleep, pentobarbital anesthesia, and (a less well defined relation) with locomotor activity. These results will be presented in that order.

Paradoxical Sleep. Table 1A gives the results at 1000 or $3200 \mathrm{~Hz}$ for paradoxical sleep and pentobarbital anesthesia. Table $1 \mathrm{~B}$ gives the results at any other frequencies which were used in paradoxical sleep, as well as some additional details. The changes with paradoxical sleep had the same time course as those described previously, (20) i.e., increasing with the first signs of hypotonia, reaching a maximum within $30-60 \mathrm{sec}$, maintaining this value until starting to decrease immediately upon awakening from paradoxical sleep, and returning to the previous level in about $10 \mathrm{sec}$ (Fig 1A). (Fig 3A starts during an episode of paradoxical sleep.) ${ }^{2}$ Because of the time needed to reach maximum, the change with paradoxical sleep is only recorded if the episode lasted longer than $30 \mathrm{sec}$.

From Table 1 the following generalities can be drawn about the impedance changes during paradoxical sleep: (a) Many or most parts of brain have impedance increases of less than $4 \%$ during paradoxical sleep; (b) The impedance increases in paradoxical sleep in the subiculum and pre-

2 Slow wave sleep or paradoxical sleep is interrupted intermittently or terminated by the rat making a movement and the EEG becoming low voltage fast which usually lasts 5 to $10 \mathrm{sec}$. I call these episodes "snuggles." The movement may be as small as moving the head for a few millimeters or as large as an extension of all legs, back, and neck, each moved 1 or $2 \mathrm{~cm}$. The rat may go back to sleep, he may lie quietly awake, or he may get up after a "snuggle." 

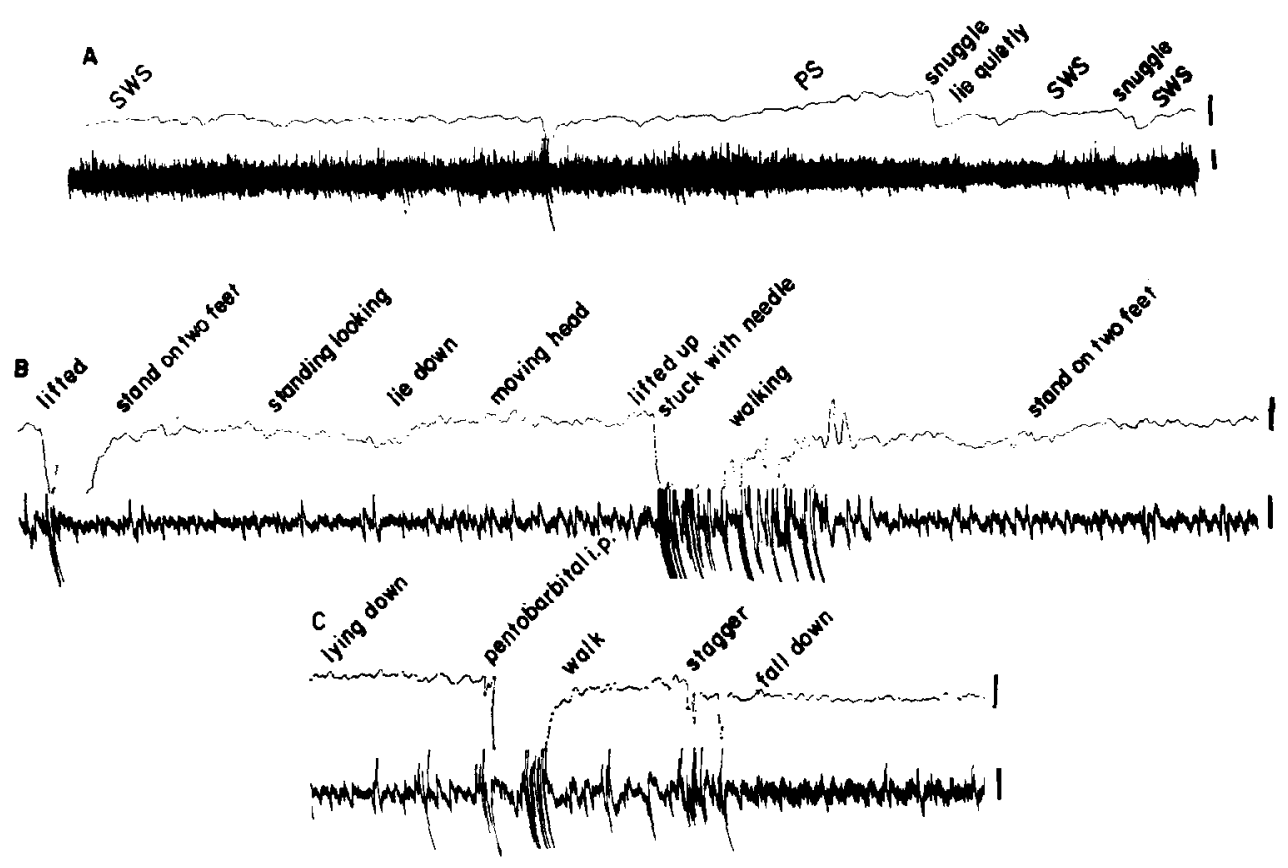

FIG. 1. A. Changes in the magnitude of impedance in slow wave sleep (SWS) and paradoxical sleep (PS). The top line is magnitude of impedance measured at $1000 \mathrm{~Hz}$, posterior configuration, (calibration marks, $5 \%$ in all). The bottom line is neocortical EEG (calibrations: $200 \mu \mathrm{v}, 60 \mathrm{sec}$ in all). Rat 107, electrode in parasubiculum and entorhinal cortex. The change at $4.9 \mathrm{~min}$ of the record during SWS is an artifact due to external interference. $B$ \& $C$. Changes in magnitude of impedance when lifted up by hand stuck with needle, and anesthetized with pentobarbital ; 30 min elapsed beween $B$ and $C$. Top line magnitude of impedance at $1000 \mathrm{~Hz}$, linear configuration. The bottom line is recording from the voltage recording electrode of the impedance electrodes. Rat 88, posterior caudate, at border of globus pallidus. Each of the three times the rat was lifted up he was removed from his shielded cage and held in an experimenter's hand, so the record is totally artifact during this time. In this and the other figures the recordings were made on curvilinear paper. None of the recordings are retouched at all, in any of the figures. In this and all other figures the label describing the behavior indicates the behavior began at the beginning of the label. That behavior continued until the next label.

$\mathrm{n}$ : The number of episodes of paradoxical sleep recorded; A: anterior configuration; P: posterior configuration; $M$ : medial configuration; Lat: lateral configuration; Lin: linear configuration; - : not done, or technically unsatisfactory; *: a previously reported animal; PS: changes in magnitude of impedance during an episode of paradoxical sleep in per cent; Pento: changes in magnitude of impedance during pentobarbital anesthesia in per cent. The designation 17 , fascia dentata and C.A4, A $1000 \mathrm{~Hz}, 2$ means that rat 17 had an electrode with tips in fascia dentata and CA4. It was run with an anterior configuration at $1000 \mathrm{~Hz}$ during two episodes of paradoxical sleep. The range of impedance changes during paradoxical sleep is in a separate column. All measurements 
during pentobarbital anesthesia were at 1000 or $3200 \mathrm{~Hz}$ but the configuration is not specified. The ventral diencephalon, midbrain, pons, and medulla are arranged in anteroposterior order. Brain stem terminology is that of Valverde (26). An increase of impedance during paradoxical sleep is measured as an amount that is sustained for ten seconds or more. Note that decreases occurred in rats 86, 83, and 144.

TABLE $1 \mathrm{~A}$

Results at 1000 or $3200 \mathrm{~Hz}$ for Paradoxical Sleep and Pentobarbital Anesthesia

\begin{tabular}{|c|c|c|c|c|c|}
\hline $\begin{array}{l}\text { Rat } \\
\text { no. }\end{array}$ & & $\begin{array}{c}\text { Configuration } \\
\text { and frequency } \\
(\mathrm{Hz})\end{array}$ & $n$ & PS $(\%)$ & Pento $(\%)$ \\
\hline \multicolumn{6}{|c|}{$\overline{\text { Ammon's horn and fascia dentata }}$} \\
\hline 17 & Fascia dentata and CA4 & A 1000 & 2 & 0 & - \\
\hline \multirow[t]{2}{*}{25} & CA3 and fornix & A 1000 & 2 & 1,2 & - \\
\hline & & $\operatorname{Lin} 1000$ & 3 & 1 & \\
\hline & $\mathrm{CA} 2$ and $\mathrm{CA} .3$ & A 1000 & 1 & 0.5 & \\
\hline & & Lin 1000 & 5 & $0-2$ & \\
\hline 31 & $\mathrm{CA} 2$ and $\mathrm{CA} 3$ & A 1000 & 1 & 0 & - \\
\hline \multirow[t]{2}{*}{76} & Basolateral amygdala and & A 1000 & 1 & 1 & -.. \\
\hline & Ammon's horn & P 1000 & 2 & $1,0.5$ & \\
\hline \multirow[t]{3}{*}{115} & CA1 and fascia dentata, & A 1000 & 3 & $0.5-2.5$ & - \\
\hline & just anterior to subicu- & $\mathrm{P} \quad 1000$ & 2 & 0,1 & \\
\hline & lum & $\operatorname{Lin} 1000$ & 2 & 0,2 & \\
\hline \multicolumn{6}{|c|}{ Subiculum and presubiculum } \\
\hline \multirow[t]{2}{*}{30} & Dorsal subiculum & A 1000 & 2 & 7,15 & -.- \\
\hline & & Lin 1000 & 1 & 2.5 & \\
\hline \multirow[t]{2}{*}{ *33 } & Dorsal presubiculum & A 1000 & 3 & $22-25$ & () \\
\hline & & Lin 1000 & 7 & $10-18$ & \\
\hline \multirow[t]{2}{*}{$* 34$} & Dorsal presubiculum & A 1000 & 6 & $5-10$ & - \\
\hline & & Lin 1000 & 7 & $5-11$ & \\
\hline \multirow[t]{2}{*}{ *35 } & Dorsal $1 / 2$ subiculum, $1 / 2$ & A 1000 & 2 & 8,10 & 一 \\
\hline & presubiculum & Lin 1000 & 52 & $7-20$ & \\
\hline \multirow[t]{2}{*}{$*_{49}$} & Dorsal subiculum & A 1000 & 17 & $10-15$ & - \\
\hline & & A 3200 & 2 & 9,11 & \\
\hline \multirow[t]{3}{*}{50} & Dorsal $2 / 3$ subiculum, $1 / 3$ & A 3200 & 3 & $7-14$ & - \\
\hline & presubiculum & $\mathrm{P} \quad 3200$ & 2 & $3.5,5$ & \\
\hline & & Lin 3200 & 30 & $12,25-30$ & \\
\hline \multirow[t]{3}{*}{111} & Ventral subiculum and pre- & M 1000 & 2 & 5,6 & - \\
\hline & subiculum & Lat 1000 & 3 & $1-3$ & \\
\hline & & $\operatorname{Lin} 1000$ & 3 & $1-4$ & \\
\hline \multirow[t]{3}{*}{112} & Ventral subiculum and sub- & M 1000 & 2 & 6 & -7 \\
\hline & arachnnid space & Lat 1000 & 28 & $3-15.5$ & \\
\hline & & Lin 1000 & 9 & $3-14$ & \\
\hline \multicolumn{6}{|c|}{ Entorhinal cortex - parasubiculum } \\
\hline \multirow[t]{2}{*}{85} & Entorhinal cortex & $\mathrm{M} \quad 1000$ & 5 & $2-4$ & - \\
\hline & & Lat 1000 & 3 & 2 & \\
\hline \multirow[t]{3}{*}{106} & Parasubiculum and ento- & M 1000 & 5 & $2-4$ & - \\
\hline & rhinal cortex & Lat 1000 & 7 & $3-10$ & \\
\hline & & Lin 1000 & 3 & $6-7$ & \\
\hline
\end{tabular}


TABLE $1 \mathrm{~A}-$ Continued

\begin{tabular}{|c|c|c|c|c|c|c|}
\hline $\begin{array}{l}\text { Rat } \\
\text { no. }\end{array}$ & & $\begin{array}{l}\text { Confir } \\
\text { and } \mathrm{fr}_{\mathrm{r}}\end{array}$ & $\begin{array}{l}\text { guration } \\
\text { requency } \\
(\mathrm{Hz})\end{array}$ & $n$ & PS $(\%)$ & Pento $(\%)$ \\
\hline 107 & $\begin{array}{l}\text { Parasubiculum and ento- } \\
\text { rhinal cortex }\end{array}$ & $\begin{array}{l}\text { M } \\
\text { La } \\
\text { Lir }\end{array}$ & $\begin{array}{l}1000 \\
1000 \\
1000\end{array}$ & $\begin{array}{l}8 \\
3 \\
3\end{array}$ & $\begin{array}{l}3-9 \\
2-5 \\
3.5-6\end{array}$ & -8 \\
\hline 108 & $\begin{array}{l}\text { Parasubiculum and ento- } \\
\text { rhinal cortex }\end{array}$ & & $\begin{array}{r}1000 \\
1000 \\
1000\end{array}$ & $\begin{array}{l}4 \\
6 \\
3\end{array}$ & $\begin{array}{l}1.5-6 \\
1-2 \\
2-5\end{array}$ & 0 \\
\hline Ventra & al diencephalon & & & & & \\
\hline 134 & $\begin{array}{l}\text { In internal capsule and glo- } \\
\text { bus pallidus, next to lateral } \\
\text { hypothalamus, preoptic } \\
\text { area, and caudate }\end{array}$ & $\mathrm{P}$ & 1000 & 3 & 0 & - \\
\hline 142 & $\begin{array}{l}\text { In internal capsule, at bor- } \\
\text { der of globus pallidus, cau- } \\
\text { date, lateral hypothala- } \\
\text { mus, and reticular nucleus } \\
\text { of thalamus }\end{array}$ & $\mathrm{A}$ & 1000 & 5 & $0-4$ & - \\
\hline 133 & $\begin{array}{l}\text { In lateral hypothalamus, } \\
\text { zona incerta, and dorso- } \\
\text { ventral nucleus of hypo- } \\
\text { thalamus, next to anterior } \\
\text { nucleus of hypothalamus }\end{array}$ & & $\begin{array}{l}1000 \\
1000 \\
1000\end{array}$ & $\begin{array}{l}4 \\
6 \\
1\end{array}$ & $\begin{array}{l}0 \\
0-4 \\
1.5\end{array}$ & 0 \\
\hline 129 & $\begin{array}{l}\text { In lateral hypothalamus, } \\
\text { median forebrain bundle, } \\
\text { field } \mathrm{H}_{2} \text { of Forel, and sub- } \\
\text { stantia nigra, less than } 0.5 \\
\text { mm from ventral surface }\end{array}$ & & $\begin{array}{l}1000 \\
1000 \\
1000\end{array}$ & $\begin{array}{l}9 \\
7 \\
4\end{array}$ & $\begin{array}{l}1-3.5 \\
1-5 \\
5\end{array}$ & -6 \\
\hline 128 & $\begin{array}{l}\text { In median forebrain bundle, } \\
\text { lateral hypothalamus, } \\
\text { ventromedial nucleus of } \\
\text { hypothalamus, zona in- } \\
\text { certa substantia nigra, } \\
\text { and fields of Forel }\end{array}$ & $\begin{array}{l}\mathrm{A} \\
\mathrm{P}\end{array}$ & $\begin{array}{l}1.000 \\
1000\end{array}$ & $\begin{array}{l}3 \\
5\end{array}$ & $\begin{array}{l}1-2.5 \\
1-3.5\end{array}$ & - \\
\hline 126 & $\begin{array}{l}\text { Zona incerta, fields of Forel, } \\
\text { and substantia nigra }\end{array}$ & $\begin{array}{l}A \\
P\end{array}$ & $\begin{array}{l}1000 \\
1000\end{array}$ & $\begin{array}{l}4 \\
3\end{array}$ & $\begin{array}{l}0 \\
0\end{array}$ & - \\
\hline 127 & Substantia nigra & $\begin{array}{l}\mathrm{A} \\
\mathrm{P}\end{array}$ & $\begin{array}{l}1000 \\
1000\end{array}$ & $\begin{array}{l}2 \\
3\end{array}$ & $\begin{array}{l}0,1 \\
1-2.5\end{array}$ & -2 \\
\hline Midbr & ain & & & & & \\
\hline 65 & $\begin{array}{l}\text { Pretectal, just touching sur- } \\
\text { face }\end{array}$ & $\begin{array}{l}\mathrm{A} \\
\mathrm{P}\end{array}$ & $\begin{array}{l}1000 \\
1000\end{array}$ & $\begin{array}{l}4 \\
2\end{array}$ & $\begin{array}{l}2-14 \\
1,3\end{array}$ & - \\
\hline 77 & $\begin{array}{l}\text { Lateral midbrain, medial } \\
\text { geniculate, and subarach- } \\
\text { noid space }\end{array}$ & $\begin{array}{l}\mathrm{A} \\
\mathrm{P}\end{array}$ & $\begin{array}{l}1000 \\
1000\end{array}$ & $\begin{array}{l}5 \\
1\end{array}$ & $\begin{array}{l}0 \\
0\end{array}$ & - \\
\hline 80 & $\begin{array}{l}\text { Superior colliculus and pre- } \\
\text { tectal area, } 1 \mathrm{~mm} \text { from } \\
\text { surface }\end{array}$ & $\begin{array}{l}\mathrm{A} \\
\mathrm{P}\end{array}$ & $\begin{array}{l}1000 \\
1000\end{array}$ & $\begin{array}{l}4 \\
1\end{array}$ & $\begin{array}{l}4-5 \\
3\end{array}$ & +4 \\
\hline
\end{tabular}


TABLE 1A-Continued

\begin{tabular}{|c|c|c|c|c|c|c|}
\hline \multicolumn{2}{|l|}{$\begin{array}{l}\text { Rat } \\
\text { no. }\end{array}$} & \multicolumn{2}{|c|}{$\begin{array}{l}\text { Configuration } \\
\text { and frequency } \\
\qquad(\mathrm{Hz})\end{array}$} & \multirow{2}{*}{$\frac{n}{2}$} & \multirow{2}{*}{$\frac{\text { PS }(\%)}{0}$} & \multirow{2}{*}{ Pento $(\%)$} \\
\hline 40 & Border of central grey and & $P$ & 1000 & & & \\
\hline & superior and inferior col- & $A$ & 3200 & 1 & 0 & \\
\hline & liculi & & 3200 & 2 & 0 & \\
\hline & & Lin & n 3200 & 1 & 0 & \\
\hline \multirow[t]{3}{*}{42} & \multirow{3}{*}{$\begin{array}{l}\text { Border of central grey and } \\
\text { superior colliculus }\end{array}$} & A & 3200 & 4 & $1.5-3$ & - \\
\hline & & & 3200 & 2 & 0,1 & \\
\hline & & Lin & 3200 & 1 & 1 & \\
\hline 43 & $\begin{array}{l}\text { Midline between superior } \\
\text { colliculi, } 1 \mathrm{~mm} \text { below sur- } \\
\text { face }\end{array}$ & $\mathrm{A}$ & 3200 & 3 & 0 & -- \\
\hline \multirow[t]{2}{*}{59} & \multirow{2}{*}{$\begin{array}{l}0.5 \mathrm{~mm} \text { below surface of } \\
\text { superior colliculus }\end{array}$} & A & 1000 & 10 & $10-18$ & \\
\hline & & $\mathrm{P}$ & 1000 & 7 & $3-7$ & - \\
\hline \multirow[t]{2}{*}{119} & \multirow{2}{*}{$\begin{array}{l}\text { Ventral tegmentum, next to } \\
\text { red nucleus }\end{array}$} & A & 1000 & 4 & $1-2$ & -- \\
\hline & & $\mathrm{P}$ & 1000 & 6 & 0 & \\
\hline 120 & $\begin{array}{l}\text { Ventral edge of central grey, } \\
\text { next to nucleus of III and } \\
\text { red nucleus }\end{array}$ & $\mathrm{A}$ & 1000 & 7 & $0-1$ & 0 \\
\hline \multirow[t]{2}{*}{137} & Lateral lemniscus, next to & A & 1000 & 4 & 0 & - \\
\hline & $\begin{array}{l}\text { lateral tegmentum under } \\
\text { inferior colliculus }\end{array}$ & $\mathrm{P}$ & 1000 & 6 & 0 & \\
\hline \multicolumn{7}{|c|}{ Pons-medulla } \\
\hline \multirow[t]{2}{*}{96} & \multirow{2}{*}{$\begin{array}{l}\text { Mid nucleus reticularis pon- } \\
\text { tis oralis, rostal part of } \\
\text { nucleus reticularis pontis } \\
\text { caudalis }\end{array}$} & A & 1000 & 5 & $0.5-1.5$ & 0 \\
\hline & & $\mathrm{P}$ & 1000 & 9 & $0-1$ & \\
\hline \multirow[t]{3}{*}{86} & \multirow{3}{*}{$\begin{array}{l}\text { Medial nucleus reticularis } \\
\text { pontis oralis }\end{array}$} & A & 1000 & 3 & $0.5,2$ & - \\
\hline & & $\mathrm{P}$ & 1000 & 3 & $-1-0$ & - \\
\hline & & Lin & 1000 & 2 & $-1-0$ & \\
\hline \multirow[t]{3}{*}{83} & \multirow{3}{*}{$\begin{array}{l}\text { Midline; mid dorsal rostal } \\
\text { pons }\end{array}$} & A & 1000 & 6 & $0.5-2$ & -2 \\
\hline & & $\mathrm{P}$ & 1000 & 3 & $-3-0$ & \\
\hline & & $\operatorname{Lin}$ & 1000 & 1 & 0 & \\
\hline \multirow[t]{3}{*}{94} & \multirow{3}{*}{$\begin{array}{l}\text { Border of griseum pontis } \\
\text { and lateral tegmentum }\end{array}$} & A & 1000 & 6 & $0-1.5$ & -3 \\
\hline & & $\mathrm{P}$ & 1000 & 4 & $0-1$ & \\
\hline & & Lin & 1000 & 1 & 2.5 & \\
\hline \multirow[t]{2}{*}{144} & \multirow{2}{*}{$\begin{array}{l}\text { In brachium conjunctivum, } \\
1 \mathrm{~mm} \text { from locus coeruleus }\end{array}$} & $\mathrm{A}$ & 1000 & 6 & $0-1.5$ & - \\
\hline & & $\mathrm{P}$ & 1000 & 3 & $-1-0.5$ & \\
\hline \multirow[t]{2}{*}{145} & \multirow{2}{*}{$\begin{array}{l}\text { On dorsal surface of brach- } \\
\text { ium conjunctivum } 0.5 \mathrm{~mm} \\
\text { from locus coeruleus }\end{array}$} & A & 1000 & 23 & $4-10$ & .. \\
\hline & & $\mathrm{P}$ & 1000 & 5 & 0 & \\
\hline \multirow[t]{2}{*}{146} & In brachium conjunctivum & A & 1000 & 3 & 0 & -1.5 \\
\hline & $\begin{array}{l}0.5 \mathrm{~mm} \text { from locus co- } \\
\text { eruleus }\end{array}$ & $P$ & 1000 & 7 & 0 & \\
\hline \multirow[t]{2}{*}{114} & \multirow{2}{*}{$\begin{array}{l}\text { Border of lateral nucleus re- } \\
\text { ticularis pontis caudalis, } \\
\text { superior olive, and nucleus } \\
\text { VII }\end{array}$} & A & 1000 & 9 & 0 & -2 \\
\hline & & $\mathrm{P}$ & 1000 & 4 & 0 & \\
\hline
\end{tabular}


TABLE $1 \mathrm{~A}-$ - Continued

\begin{tabular}{|c|c|c|c|c|c|c|}
\hline \multirow{3}{*}{$\frac{\begin{array}{c}\text { Rat } \\
\text { no. }\end{array}}{104}$} & \multirow{3}{*}{$\begin{array}{l}\text { Border of lateral nucleus } \\
\text { reticularis pontis caudalis, } \\
\text { superior olive, and nucleus } \\
\text { VIII }\end{array}$} & \multicolumn{2}{|c|}{$\begin{array}{l}\text { Configuration } \\
\text { and frequency } \\
\qquad(\mathrm{Hz})\end{array}$} & \multirow{3}{*}{$\begin{array}{l}n \\
1 \\
5\end{array}$} & \multirow{3}{*}{ 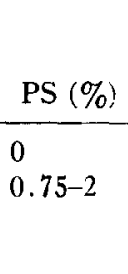 } & \multirow{3}{*}{$\frac{\text { Pento }(\%)}{-}$} \\
\hline & & A & 1000 & & & \\
\hline & & $\mathrm{P}$ & 1000 & & & \\
\hline \multirow[t]{2}{*}{122} & \multirow{2}{*}{$\begin{array}{l}\text { Lateral tegmentum of rostal } \\
\text { medulla }\end{array}$} & A & 1000 & 3 & 0 & \multirow[t]{2}{*}{0} \\
\hline & & $\mathrm{P}$ & 1000 & 6 & 0 & \\
\hline \multirow[t]{2}{*}{110} & In vestibular nuclei, less than & A & 1000 & 10 & $4-9$ & \multirow[t]{2}{*}{-6} \\
\hline & $0.5 \mathrm{~mm}$ below surface & $\mathrm{P}$ & 1000 & 5 & $2-3$ & \\
\hline \multirow[t]{2}{*}{117} & \multirow{2}{*}{ Midline mid rostal medulla } & A & 1000 & 2 & 1,2 & \multirow[t]{2}{*}{ - } \\
\hline & & $\mathrm{P}$ & 1000 & 2 & 0 & \\
\hline \multirow[t]{2}{*}{118} & \multirow[t]{2}{*}{ Midline ventral medulla } & A & 1000 & 4 & $2-4$ & \multirow[t]{2}{*}{-} \\
\hline & & $P$ & 1000 & 5 & 0 & \\
\hline \multirow[t]{3}{*}{121} & \multirow[t]{3}{*}{ Lateral ventral medulla } & $\mathrm{A}$ & 1000 & 5 & $0-2$ & \multirow[t]{3}{*}{ 一 } \\
\hline & & $\mathrm{P}$ & 1000 & 3 & $0-3$ & \\
\hline & & Lin & 1000 & 4 & $0-2$ & \\
\hline 123 & Midline mid rostal medulla & A & 1000 & 6 & $1-2$ & 0 \\
\hline \multirow[t]{2}{*}{124} & \multirow{2}{*}{$\begin{array}{l}\text { Midline just above pyramids, } \\
\text { in rostal medulla and cau- } \\
\text { dal pons }\end{array}$} & A & 1000 & 3 & $1-1.5$ & \multirow[t]{2}{*}{ - } \\
\hline & & $\mathrm{P}$ & 1000 & 5 & $0-2$ & \\
\hline \multicolumn{7}{|c|}{ Other sites } \\
\hline \multirow[t]{2}{*}{67} & \multirow[t]{2}{*}{ Neocortex } & A & 1000 & 1 & 0 & \multirow[t]{2}{*}{0} \\
\hline & & $\mathrm{P}$ & 1000 & 2 & $0,1.5$ & \\
\hline \multirow[t]{2}{*}{69} & Neocortex & A & 1000 & 2 & 1 & -1.5 \\
\hline & & $\mathrm{P}$ & 1000 & 4 & 1 & \\
\hline 71 & Neocortex & A & 1000 & 3 & $0.5-1$ & - \\
\hline 88 & Posterior caudate, at border & A & 1000 & 3 & 0.5 & -3 \\
\hline & of globus pallidus & $\mathrm{P}$ & 1000 & 3 & 0 & \\
\hline 89 & Caudate & A & 1000 & 3 & 1 & -1.5 \\
\hline & & $\mathrm{P}$ & 1000 & 3 & 1 & \\
\hline 64 & $\begin{array}{l}\text { At border of caudate, inter- } \\
\text { nal capsule, fornix and } \\
\text { lateral ventricle }\end{array}$ & A & 1000 & 16 & $3-8$ & +2 \\
\hline 72 & Piriform cortex & A & 1000 & 2 & 1,2 & - \\
\hline 74 & Reticular nucleus of thala- & A & 1000 & 2 & 0 & - \\
\hline & mus & $\mathrm{P}$ & 1000 & 3 & 0 & \\
\hline 75 & Lateral nucleus of thalamus & A & 1000 & 4 & $2-3$ & -1.5 \\
\hline & & $\mathrm{P}$ & 1000 & 6 & $0-0.5$ & \\
\hline 113 & Septum & $\mathrm{A}$ & 1000 & 7 & $0-4$ & - \\
\hline & & $\mathrm{P}$ & 1000 & 5 & $0-3$ & \\
\hline
\end{tabular}

subiculum are the largest seen, usually well over $10 \%$ and up to $25-30 \%$; (c) The impedance increases in entorhinal cortex and parasubiculum in paradoxical sleep are of intermediate value-usually 3-10\%; (d) Decreases in impedance in paradoxical sleep were seen in only three sitesthe posterior configurations of rats 86,83 , and 144 , all in the pons. The 
decrease did not occur in all episodes but when it did occur it was unequivocal; (e) There may be large increases in the vicinity of the pretectal area; and (f) All sites with increases greater than $4 \%$ were within $1 \mathrm{~mm}$ of a pial or ependymal surface.

The only other rats with increases greater than $4 \%$ are $129,65,80,59$, 145,110 , and 64 . Of these all but 80 had some electrode tips just touching a surface or within $0.5 \mathrm{~mm}$ of a surface and 80 was $1 \mathrm{~mm}$ from a surface. I do not know how to evaluate the fact that these tips are near the surface, but it will be discussed below. The electrode tips in rats 65,80 , and 59 are all in the vicinity of the pretectal area. Since two of these had electrodes very close to the surface ( 65 and 59 ), and one (65) had a small brain abscess, although at a distance from the electrode tips, I am less sure of impedance changes in the vicinity of the pretectal area than of the other changes.

The impedance changes during paradoxical sleep at other frequencies in Table $1 \mathrm{~B}$ show that in general similar changes occur at these other frequencies, although most of these changes are too small for careful comparison.

Pentobarbital Anesthesia. The impedance changes with pentobarbital anesthesia occurred over a 2 - to 10 -min period as the rat became anesthetized (Fig $1 \mathrm{~B}$ and $\mathrm{C}$ ). Of the 23 measurements only two were increases, eight showed no change, and 13 were decreases (Table 1A). There

\section{TABLE 1 B}

Impedance Changes During Paradoxical Sleep at Other Frequencies and Other Details

The designation $17, \mathrm{~A} 32 \mathrm{~Hz}, 8 ; 0 \%$ : means that rat 17 was run with an anterior configuration at $32 \mathrm{~Hz}$. Eight episodes of paradoxical sleep were seen, and the impedance change was zero. The anatomical site of 17 is identified in Table $1 \mathrm{~A}$.

17. A $32 \mathrm{~Hz}, 8 ; 0 \%$

Ammon's horn-fascia dentata

25 , A $32 \mathrm{~Hz}, 1 ; 1 \%$

26 , Lin $32 \mathrm{~Hz}, 1 ; 1 \%$

31, A $32 \mathrm{~Hz}, 1 ; 0 \%$

31 , Lin $32 \mathrm{~Hz}, 1 ; 0 \%$

76, P $32 \mathrm{~Hz}, 1$; $1 \%$

76, P $10,000 \mathrm{~Hz}, 1 ; 2 \%$

30 , Lin $32 \mathrm{~Hz}, 3 ; 2-5 \%$

Subiculum-presubiculum

*33, A $32 \mathrm{~Hz}, 3 ; 20-26 \%$

*33, Lin $32 \mathrm{~Hz}, 4 ; 10-12 \%$

*34, A $32 \mathrm{~Hz}, 5 ; 5-7 \%$

*34, Lin $32 \mathrm{~Hz}, 5 ; 4-9 \%$

*34, A $10,000 \mathrm{~Hz}, 1$; $4 \%$ 
TABLE 1B-Continued

$* 34$, Lin $10,000 \mathrm{~Hz}, 2 ; 5 \%, 6 \%$

*35, A $32 \mathrm{~Hz}, 3 ; 6-8 \%$

*35, Lin $32 \mathrm{~Hz}, 3 ; 5-8 \%$

*35, A $10,000 \mathrm{~Hz} ; 9 \%$

*49, A $32 \mathrm{~Hz}, 4 ; 10-14 \%$

*49, A $100 \mathrm{~Hz}, 1 ; 14 \%$

*49, A $32 \mathrm{~Hz}, 1 ; 14 \%$

$50, \mathrm{P} 32 \mathrm{~Hz}, 1 ; 10 \%$

112, This rat was very sick. Many of the episodes at lateral and linear configurations are less than $30 \mathrm{sec}$ in duration, which $\mathrm{I}$ have seen in no other rats. These data for less than $30 \mathrm{sec}$ episodes are included in the data of Table $1 \mathrm{~A}$.

Entorhinal cortex-parasubiculum

107, M $10,000 \mathrm{~Hz}, 3 ; 0-2 \%$

107 , Lat $10,000 \mathrm{~Hz}, 2 ; 3 \%, 4 \%$

Ventral diencephalon

129, A $32 \mathrm{~Hz}, 2 ; 5 \%, 6 \%$

\section{Midbrain}

40, A $32 \mathrm{~Hz}, 1 ; 0 \%$

$42, \mathrm{P} 32 \mathrm{~Hz}, 1 ; 1 \frac{1}{2} \%$

59 , A $32 \mathrm{~Hz}, 22 ; 3-18 \%$

59, P $32 \mathrm{~Hz}, 8 ; 4-12 \%$

59 , A $10,000 \mathrm{~Hz}, 1 ; 9 \%$

$65, \mathrm{P} 32 \mathrm{~Hz}, 3 ; 4 \%$. There is an abscess nearby and the brain is somewhat distorted.

86, P $32 \mathrm{~Hz}, 1$; decrease $1 \frac{1}{2} \%$

$$
\text { Pons-medulla }
$$

$86, \mathrm{P} 10,000 \mathrm{~Hz}, 2$; decrease $0 \%, 1 \%$

83 , A $32 \mathrm{~Hz}, 3 ; 0-2 \%$

83, P $32 \mathrm{~Hz}, 3$; decrease $0-3 \%$

83 , A 10,$0003 ; 0.5-1.5 \%$

$83, \mathrm{P} 10,000,1 ; 0.5 \%$

94, A $32 \mathrm{~Hz}, 3 ; 0-4 \%$

94, P $32 \mathrm{~Hz}, 4 ; 0 \%$

$94, \mathrm{P} 10,000 \mathrm{~Hz}, 2 ; 1 \%$

94, Lin $10,000 \mathrm{~Hz}, 2 ; 1 \%$

145, A $32 \mathrm{~Hz}, 4 ; 6-7 \%$

145 , A $10,000 \mathrm{~Hz}, 10 ; 4-6 \%$

110, A $32 \mathrm{~Hz}, 5 ; 0-3 \%$

110 , A $10,000 \mathrm{~Hz}, 5 ; 3-10 \%$

$67, \mathrm{P} 32 \mathrm{~Hz}, 3 ; 0 \%$

Other sites

69, P $32 \mathrm{~Hz}, 1 ; 3 \%$

89 , A $32 \mathrm{~Hz}, 3 ; 0-1.5 \%$

89, P $32 \mathrm{~Hz}, 3 ; 0 \%$

89 , A $10,000,1 ; 0 \%$

64 , A $32 \mathrm{~Hz}, 6$; 5-11\%

64, A $10,000 \mathrm{~Hz}, 4 ; 1-4 \%$

75 , A $10,000 \mathrm{~Hz}, 2 ; 0-1.5 \%$ 

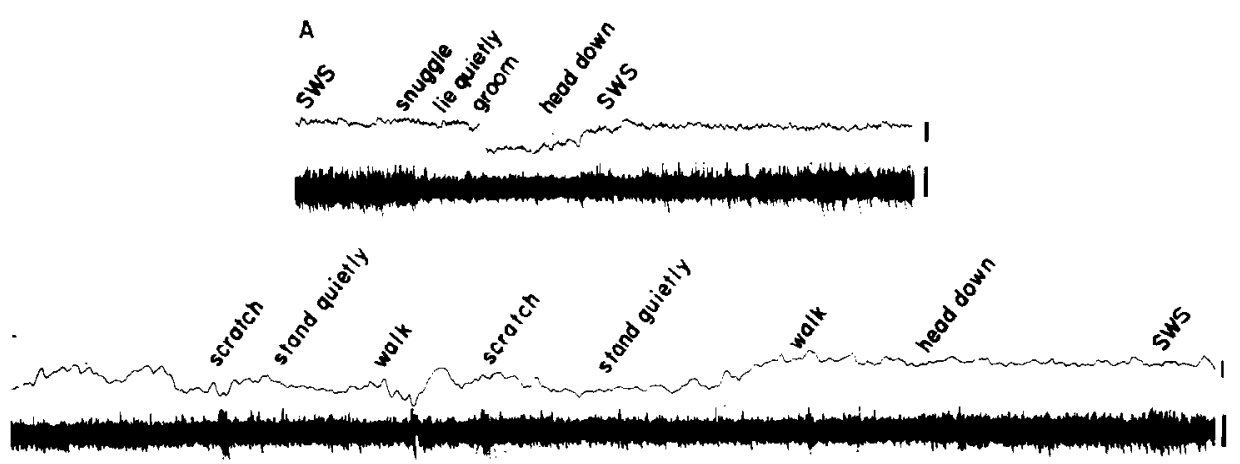

FIG. 2A and B. During activity. The top line is magnitude of impedance A: 10,000 $\mathrm{Hz}$ anterior configuration. $\mathrm{B}: 1000 \mathrm{~Hz}$ anterior configuration. The bottom line is neocortical EEG. Rat 107, parasubiculum and cotorininal cortex. Calibrations as in Fig. 1.

does not appear to be any relation between these changes and location in brain or the changes in paradoxical sleep. This measurement was made while the rats were being anesthetized in preparation for perfusion fixation. Most of these measurements were technically unsatisfactory and are not reported in Table $1 \mathrm{~A}$ because the major concern was to get a good perfusion. The impression from these technically unsatisfactory data is in agreement with the good data, that in most places there is a decrease in impedance with anesthesia.

Activity. When the rats were awake, changes in magnitude of impedance were often seen, some as much as $10 \%$, usually less than $3 \%$, and almost all these changes were decreases. The best behavioral correlation which I could find with these changes was with motor activity. These changes were either an increased variability or a generalized decrease, and in some cases these two types of changes occurred simultaneously. Figure $2 \mathrm{~A}$ shows an example of a decrease of impedance when a rat who had previously been inactive started to groom himself. Theimpedance did not decrease when the rat "snuggled" and awakened but was inactive. Then as he began motor activity (grooming), the impedance decreased $7 \%$ within $3 \mathrm{sec}$. (The time constant of averaging here is $0.1 \mathrm{sec}$, so this ratc of change is the true onc.) The impedance then remained decreased during the grooming, started to increase when the rat lay down again and became inactive, and returned to the baseline value in about $35 \mathrm{sec}$. The onset was the most rapid change seen. Most changes had a time course more like the return to baseline. This example was also atypical in that there was such a clear-cut relation to activity, but other clear examples did occur.

Figure $3 \mathrm{~B}$ is an example of increased variability of the impedance during activity. There was a general increase of impedance during drinking, but 


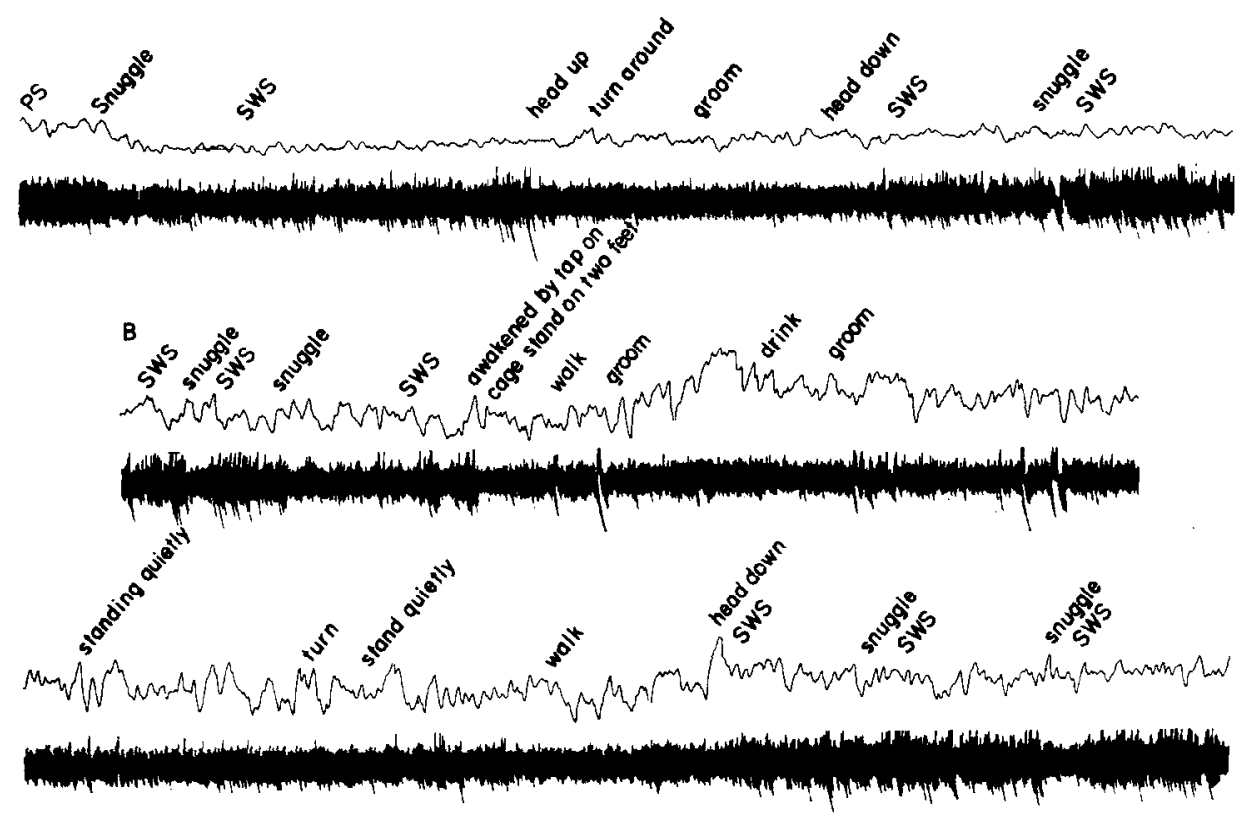

FIG. 3A and B. During activity. The record in A starts during an episode of PS. Top line magnitude of impedance at $1000 \mathrm{~Hz}$. A : anterior configuration, B : Posterior configuration. The bottom line is neocortical EEG. The recording in B is continuous. Rat 119 , ventral tegmentum next to red nucleus. Calibrations as in Fig. 1.

for most of the activity the average level of impedance did not change although the variability did. In the detailed description of the impedance measuring system previously published, it was shown that the measured impedance was independent of other electrical activity in the voltage recording electrodes of the impedance electrodes. This was checked further in these studies. Records of the electrical activity from the voltage recording electrodes showed that there is not necessarily an increased electrical activity during activity whether real or artifact, so this increased variability was not always due to increased "noise." This is shown in Fig. 1B and C when the lower line is recording from the voltage recording electrodes of the impedance electrodes. Movement artifact and artifact due to lifting out of the cage were present, and when anesthetized the slow wave amplitude was larger. But many of the decrcascs were unrelated to this increased noise. Note however, that large artifacts such as from lifting out of the cage did cause artifactual changes in impedance. This noise level has been checked at all sites during activity. I can see no relation between the fluctuations of impedance during activity and any particular kind of activity. Rates of change of up to $3 \%$ in $3 \mathrm{sec}$ were seen (Fig. 3B). (Since the time 
constant of averaging in this case is $1.0 \mathrm{sec}$, there may be attenuation of the rate of change by the averaging process here.) (The impedance during the slow wave sleep at the beginning of the record was $2 \%$ less than that during slow wave sleep at the end of the record 11 min later. Slow changes of this sort were common. I have been able to see no pattern to them.)

Figure $2 \mathrm{~B}$ is an example in which there is a general decrease of impe. dance during much of the activity of about $6 \%$ but also some greater fluctuations. However, in this case when the animal walks, about $15 \mathrm{sec}$ before going into slow wave sleep, at the end of the record, there was no change of impedance.

Figure $3 \mathrm{~A}$ is an example of no change during activity, except for a small increase in variability. [Fig. 9C of $(20)$ is another example of this.] (In the second episode of slow wave sleep in Fig. 3A the impedance was $2 \%$ greater than in the first.)

All the records of Fig. 2 and Fig. 1A are from the same rat and all of those in Fig. 3 from another rat, to stress the point that I have not been able to identify a constant pattern of response during activity even at the same site. Some generalizations about different sites do emerge, though Table 2 gives a very simple description for each site. Sites in entorhinal cortex and brain stem seem to be those which most commonly gave the largest changes with activity, but there were many exceptions and no finer anatomical pattern was apparent. Rats 88 and 89 with electrode tips in the caudate nucleus were the two which showed almost no changes other than with paradoxical sleep and anesthesia. Impedance in Ammon's horn, fascia dentata, subiculum, and presubiculum sites usually changed less than $2 \%$ with activity and any changes were less common than at most other sites. Changes in neocortex, while present, were usually less than $1 \%$.

In describing these data I have spoken of the correlation with activity, but have pointed out some exceptions. There seems to be a better correlation with activity than with arousal, orienting, or attending to the environment, but serious attempts were not made to find the best behavioral correlate of the changes.

These data take on particular interest in view of the reports from the Adey-Kado group on impedance changes in various behavioral states. They $(2,5)$, working on cats, reported (a) a $2-3 \%$ decrease in resistive impedance with arousing physiological stimuli in the Ammon's horn; and (b) a $1.5 \%$ increase in resistive impedance in transition from wakefulness to slow wave sleep in hippocampus and septum and amygdala. In measurements in comparable situations I cannot corroborate these findings. The Adey-Kado group also reported changes of up to $15 \%$ during various stages of learning a t-maze performance in hippocampus, amygdala, and midbrain reticular formation $(2-5)$. I have no camparable data, but have 
TABLE 2

Changes in Magnitude of Impedance During Motor Activity The location of the electrode tips in each rat is given in Table $1 \mathrm{~A}$.

17 technically unsatisfactory

25 decrease $1-2 \%$, many exceptions

26 usually no change, some decreases to $4 \%$

31 usually no change, constant generally

76 decrease $1-3 \%$, constant generally

115 decrease $0-2 \%$

Subiculum-presubiculum

30 no consistent change

33 usually no change, constant generally

34 usually no change, constant generally

35 usually no change, constant generally

49 usually no change, constant generally

50 usually no change

111 decrease $0-3 \%$

112 decrease $0-3 \%$

Entorhinal cortex-parasubiculum

85 usually no change, constant generally

106 decrease $0-10 \%$

107 decrease $1.5-10 \%$, usually $3-4 \%$

108 decrease $2-7 \%$

Ventral diencephalon

134 usually no change

142 some increase $1 \%$, some decrease to $3 \%$

133 usually no change

129 some increase $1-2 \%$, some decrease $1-2 \%$

128 decrease $0-2 \%$, decrease $5 \%$ with prolonged activity

126 some increase to $7 \%$, some decrease to $7 \%$

127 decrease $0-2 \%$, usually no change

Midbrain

65 decrease $1-3 \%$

77 technically unsatisfactory

$800-6 \%$ increase

40 consistently decreases up to $10 \%$

42 occasional decrease

43 technically unsatisfactory, no clear relation

59 consistently decreases $5-10 \%$

119 decrease $1-2 \%$ usually

120 decrease $0-1 \%$

137 usually no change

\section{Pons-medulla}

96 usually no change

86 decrease $2 \%$

83 no correlation, some increase up to $5 \%$, some decrease down to $4 \%$

94 usually no change, once decrease $4 \%$

144 usually no change

145 no clear change, drifty record 
TABLE 2-Continued

\section{6 usually no change}

\section{Pons-medulla}

114 no correlation, some increase to $1 \%$, some decrease to $1 \%$

104 no correlation, some increase $1 \frac{1}{2} \%$, some decrease to $21 \% 2 \%$

122 decrease $0-3 \%$

110 decrease $1.5-51 / 2 \%$

117 usually no change

118 usually no change, some decrease $1 \%$

121 usually no change

123 decrease $0-2 \%$, constant generally

124 technically poor

Other sites

67 (neocortex) decrease $0-1 \%$, constant generally

69 (neocortex) usually no change

71 (neocortex) decreases $0-0.5$, constant generally

88 (caudate) usually no change, constant generally

89 (caudate) usually no change, constant generally

64 (ventricle) usually decrease, $1 \%$ increase to $5.5 \%$ decrease

72 (piriform cortex) usually no change

74 (thalamus) usually no change

75 (thalamus) usually no change, constant generally

113 (septum) consistently derrease $1 \%$ to $3 \%$

seen changes in many parts of brain of comparable magnitude while rats were awake and active. This will be further discussed below.

No changes were seen in the transition between slow wave sleep and quiet arousal. When the rat attended to some external stimuli, for instance, a noise or a movement of the observer, there were usually no changes in impedance. In only a few cases a correlation between theta activity in the hippocampus during attentive behavior and impedance changes in hippocampus was looked for and none was seen. No tests requiring discriminative performance were given. While the rats were watched as they went through their repertoire of behavior, all of this occurred in a cage with which they were thoroughly familiar (in order to facilitate sleeping), so that there were no observations made while the rat was problem solving, facing a novel stimulus or situation, or under conditions of high drive state.

While the basic method would allow it, it is not possible with the present apparatus to measure impedance from more than one testing frequency at the same time or to measure both magnitude and phase at the same time. None of the changes seen in an awake rat were consistent enough to allow measurements made at different times to be compared. Therefore, in almost all cases, measurements were made of magnitude of impedance at $1000 \mathrm{~Hz}$ only. 
No other impedance changes were seen other than in these three states, except for slow changes over many minutes, such as those illustrated in Fig. $3 \mathrm{~A}$ and $\mathrm{B}$ and as mentioned above.

\section{Discussion}

Paradoxical Sleep and Anesthesia. One possibility for the increase of impedance with paradoxical sleep may seem to be that the brain is moving relative to the electrode. However, this cannot be true because the impedance decreases only slightly at three sites during paradoxical sleep, and there should be roughly equal numbers of sites with decreases as with increases if the change were due to motion. To be sure that the large changes in subiculum and presubiculum were not due to movement, rats 111 and 112 had an electrode placed in the ventral subiculum so the body of the electrode, which supports the electrode tips, entered the subiculum and presubicuium with the opposite orientation from those electrodes placed in the dorsal subiculum and presubiculum. Similar increases occurred in both groups. Other evidence against movement of brain is that many workers, including myself, have recorded from single cells during paradoxical sleep and are able to hold the cells (11).

All the electrode locations which showed impedance increases with paradoxical sleep of greater than $4 \%$ were within $1 \mathrm{~mm}$ of an ependymal or pial surface of brain-all the stibiculum-presubiculum group, all the entorhinal cortex-parasubiculum group, and rats 129, 65, 80, 59, 145, 110, and 64 . Many of these were within $0.5 \mathrm{~mm}$. However, in rats 77 and 124, electrode tips were within $0.5 \mathrm{~mm}$ of a surface and the changes were less than $4 \%$. Note that entorhinal cortex, parasubiculum, presubiculum, and subiculum have a pial surface, wheras Ammon's horn does not, and there are only very small impedance changes in Ammon's horn. If an electrode tip was just barely touching or dimpling a surface, as was the case for $112,65,77$, 145 , this might make the recorded impedance especially sensitive to very small movements, but I cannot see how a tip in the substance of brain even though near the surface could lead to artifact. All the electrodes enter from the dorsal surface, so in some the surface was pierced by the electrodes; in others the surface was not. There is no relation between impedance changes in activity and surfaces. This surface effect is thus not artifact.

Bennett (6) has recently shown unexpectedly large impedances across an arachnoid surface (which he shows is what has been often called "pial" surface). He also showed large resistance across the ependyma of choroid plexus but not over the alveus of the hippocampus, in agreement with known anatomy of junctions between ependymal cells. I know of no data to suggest that resistances at these surfaces might change; however, the 
relation of impedances changes in paradoxical sleep to surfaces is so strik ing that changes in these surface impedances much be considered. If all the tips are on the same side of the surface, as they are in all except rats 112 77 , and 64 (and possibly in 65 and 145), then much more current shoulc flow across the surface in a radial than in a linear configuration. But im pedance changes in paradoxical sleep are not greater in radial than lineat configurations, so I think that it is unlikely that changes in surface impe dance are the cause of these measured impedance changes.

Adey, Kado, and Walter (5) have noted a $0.3 \%$ increase in impedance in the amygdala during paradoxical sleep. Birzis and Tachibana (7) re corded changes in impedance pulses in paradoxical sleep and arousal. Thesf are pulses of less than $1 \%$ for less than $0.5 \mathrm{sec}$ and are assumed to be due to blood flow and are clearly not related to slow changes recorded in $\mathrm{my}$ studies or by the Adey-Kado group.

There is an increase of electrical impedance of brain during seizure activ ity of about $5 \%$, sometimes up to $10-20 \%(9,29)$. Van Harreveld and Schade (29) suggested that this is due to $\mathrm{NaCl}$ leaving interstitial space and entering cells associated with the increased activity. Fertziger and Ranck (10) have developed this idea further in terms of the potassium accumulation in the interstitial space which occurs in seizures, somewhat similar to that proposed for spreading depression (19). In paradoxical sleep there is an increase in the rate of firing of neurons in almost all reported cases (11). However, this increase is less than that in a seizure For instance, blood flow increases over three and a half times in a seizure (17) and $80 \%$ in paradoxical sleep in cats (23), and blood flow may well be a monotonically increasing function of neural activity. "Multiple unit" activity in some sites in brain decreases during anesthesia (7a). Since blood flow is $60 \%$ of the normal (12), there is strong suggestion that there is a generalized decrease. Many workers have the impression that anesthesia decreases spontaneous firing of cells from experience with unanesthetized acute preparations. It is thus tempting to think that the decrease of impedance with anesthesia may be due to the same mechanism that increases it in paradoxical sleep and in seizures and reflects the average rate of firing of neurons in an area. In this light it should be noted that there was no correlation seen between the occurrence of spikes in the spontaneous electrical activity recorded at appropriate pontine locations during paradoxical sleep and impedance changes.

As a follow up of the previous findings of large impedance increases in the subiculum and presubiculum in paradoxical sleep (20), these findings of much smaller changes elsewhere are disappointing. However, the lack of large changes elsewhere makes the large changes in subiculum and presubiculum and moderate sized ones in parasubiculum and entorhinal cortex 
of even more interest. At present there are no data to indicate the basis for these changes. Indeed, the only other experiments in which changes in these different parts of the hippocampal formation were examined during paradoxical sleep (21) do not show these parts as clearly different from Ammon's horn and the fascia dentata.

Activity. The possibility that the impedance changes with activity are due to movement of the brain referent to the electrode must be considered. The evidence against this possibility is similar to that against the possibility for the changes in paradoxical sleep. Most of the changes are decreases. If they were due to movement, some would be increases and some decreases. Others $(15,16)$ and I have been able to record with a microelectrode from a single neuron in many of these regions during movements of rats. The electronic system had a small amount of movement artifact in it, giving changes of a few per cent, but only with movements more violent than those which occurred in these rats. Even much milder shaking of the wires was not sustained by the rat.

The two instances in which I could not corroborate the results of the Adey-Kado group were among the earlier of their reports and among the least thoroughly studied of their results. Their most striking results are decreases in impedance with the initiation of a discrimination (walking down a runway) at a high level of performance (4). This may well be the same or similar to the decreases I see with "activity." The inconsistency I see in relation to activity may reflect the equivalent of "level of performance" in an open field situation. In any event large impedance changes do occur when an awake animal is doing things.

The impedance method of the Adey-Kado group is a two-electrode system-the same electrodes pass current as record the voltage. A four-electrode method used in this study avoids many of the problems of the electrode-brain interface and current flowing through damaged brain. There would seem to be little significant conflict between the results of these two methods and, whereas most results are not directly comparable, there is a general agreement that changes of comparable magnitude do occur. Changes seem to be much more widespread than previously imagined.

In an earlier paper the magnitude and phase angle of impedance changes in subiculum and presubiculum during paradoxical sleep were reported at various testing frequencies (20). From these fairly complete data it was possible to discuss with some detail just what the possible mechanisms for this impedance change could be. The only attractive possibilities were a change in some low resistance connection between cells or a change in resistance of interstilial space, with the latter seeming most likely. None of the changes in paradoxical sleep reported here were as fully studied as those in the previously reported group, but the results at different fre- 
quencies are similar, so that the reasoning in these previous cases seem very likely to hold in all the changes in paradoxical sleep. The data for anesthesia and activity are not complete enough to allow a decision to be made.

A recent paper by Cole, $\mathrm{Li}$, and Bak (8) discusses the use of the Maxwell and Raleigh equations in analyzing impedance measurements in tissue. They say, "At low frequencies of applied current, and for cells of small dimensions in the direction of the applied field, mombrane conductance is usually so low that the cells may be considered as nonconductors. . . Under these conditions the equations derived by Maxwell and Raleigh have often been applied to give a measure of the volume fraction occupied by cells...." This is correct. However, in the nervous system where fibers, dendrites, and glial extensions are long and narrow, the cells are not of small dimensions in the direction of the applied field in grey matter where there are some of these elements within any given orientation. In white matter it can only be applied in a tract where all the fibers are going in one direction, and then only in a direction normal to the orientation of the fibers. Nevertheless these workers apply the Maxwell equation to gray matter, which is an error by their own standards. This might be considered minor, except that the stature of the authors is such that this error might be repeated with some confidence by others. The uses of the Maxwell and Raleigh equations in the nervous system are very limited and the major use is in tissues other than nervous system. The fact that this paper was published in a journal dealing with the nervous system might well mislead other workers in ways which the authors did not intend.

As I have argued in some detail earlier (18), if the frequency-dependent length constant of fibers or dendrites or glial extensions is less than the length of the element and less than the separation of current electrodes used in a given method, then a significant amount of current will flow in that element if it has a similar orientation to that of the current. Two studies have since shown that in current flow in white matter more current indeed flows in the cytoplasn of the myelinated fibers than in the interstitial space when the field is in the same orientation as the fiber $(14,22)$. Therefore, when impedance changes are seen in brain, the possibility that this is due to some change in current flow inside cells must be considered. The existence of low resistance connection between neurolglia cells in amphibia and leaches (13) and between mammalian neuroglia cells and neurons in tissue culture (30) gives this possibility more than theoretical significance.

There are two cases in which the cause of impedance increases is clear. asphyxia (27) and spreading depression (28), and in both there is a decrease in the size of interstitial space. In the other cases of impedance in- 
crease, changes in the interstitial surface seem most likely, and indeed there are many suggestions that this is the case and why it is the case (1, $25,31)$. Nevertheless, with the two exceptions, impedance changes remain unexplained phenomena. The magnitude of many of these changes and the suggestions of its basis suggest that it is reflecting some important things in the nervous system beyond the scope of much of our current understanding.

The largest impedance changes seen in normal functioning are in the subiculum and presubiculum in paradoxical sleep. Since paradoxical sleep is such a stereotyped response occurring in well defined episodes, this would seem to be a particularly useful situation in which to investigate the nature of the impedance changes.

\section{References}

1. Adey, W. R., B. G. Bystrom, A. Costin, R. T. Kado, and T. J. Takby. 1969. Divalent cations in cerebral impedance and cell membrane morphology. Exp. Neurol. 23 : 29-50.

2. Adey, W. R., R. T. Kano, and J. Diuro. 1962. Impedance measurements in brain tissue of animals using microvolt signals. Exp. Neurol. 5: 47-66.

3. Adey, W. R., R. T. Kado, J. Didro, and W. J. Schindler. 1963. Impedance changes in cerebral tissue accompanying a learned discriminative performance in the cat. Exp. Neurol. $7: 259-281$.

4. Adey, W. R., R. T. Kaido, J. T. McIlwain, and D. O. Walter. 1966. The role of neuronal elements in regional cerebral impedance changes in alerting, orienting, and discriminative responses. Exp. Neurol. $15: 490-510$.

5. ADEY, W. R., R. T. KADO, and D. O. WALTER. 1965. Impedance characteristics of cortical and subcortical structures: evaluation of regional specificity in hypercapnia and hypothermia. Exp. Neurol. 11 : 190-216.

6. Bennett, M. V. L. 1969. Electrical impedance of brain surfaces. Brain Res. 15: $584-590$.

7. Birzis, L., and S. Tachibana. 1964. Local cerebral impedance and blood flow during sleep and arousal. Exp. Neurol. 9: 269-285.

7a. Buchwald, J. S., D. S. Weber, S. B. Holstein, F. S. Grover, and J. A. SchWAFr. 196). Quantified unit background activity in the waking cat during paraiysis, anesthesia, and cochlear destruction. Brain Research. 15 : 465-482.

8. COLE, K. S., C..L. LI, and A. F. BAK. 1969. Electrical analogues for tissues. Exp. Neurol. 24: 459-473

9. Elazar, Z., R. T. Kano, and W. R. Adey. 1966. Impedance changes during epileptic seizures. Epilepsia $7: 291-307$.

10. Fertziger, A. P., and J. B. Ranck, Jr. 1970. Potassium accumulation in interstitial space during epileptiform seizures. Exp. Neurol 26, In Press.

11. Jouver, M. 1967. Neurophysiology of the states of sleep. Physiol. Kev. 47: 117177.

12. KetY, S. S. 1967. Relationship between energy metabolism of the brain and functional activity. Res Publ. Ass. Nerv. Ment. Dis. 45 : 39-45.

13. Kuffler, S. W., J. G. Nicholls, and R. Orkand. 1966. Physiological properties of glial cells in the central nervous system of amphibia. J. Neurophysiol. 29 : 768-787. 
14. Nicholson, P. W. 1965. Specific impedance of cerebral white matter Exp. Neurol 13 : $386-401$.

15. Noda, H., S. Manohar, and W. R. Adey. 1969. Spontaneous activity of cat hippocampal neurons in sleep and wakefulness. Exp. Neurol. 24 : 217-231.

16. Olds, J., W. D. Mink, and P. J. Best. 1969. Single unit patterns during anticipatory hehavior. Elcctrocnccphalogr. Clin. Ncurophysiol. 26 : 144-158.

17. Plum, G., J. B. Posner, and B. Troy. 1968. Cerebral metabolic and circulatory responses to induced convulsion in animals. Arch. Neurol. 18: 1-13.

18. Ranck, J. B., JR. 1963. Analysis of specific impedance of rabbit cerebral cortex. Exp. Neurol. 7 : 153-174.

19. RANCK, J. B., JR. 1964. Specific impedance of cerebral cortex during spreading depression, and an analysis of neuronal, neuroglial, and interstitial contributions. Exp. Ncurol. 9: 1-16.

20. Ranck, J. B., JR. 1966. Electrical impedance in the subicular area of rats during paradoxical sleep. Exp. Ncurol. $16: 416-437$.

21. RANCK, J. B., JR. 1969. Macroelectrode responses with stimulation and recording within hippocampus and related structures during different states of consciousness. Exp. Neurol. 23 : 207-213.

22. Ranck, J. B., JR., and S. L. BeMent, 1965. The specific impedance of the dorsal columns of cat: an anisotropic medium. Exp. Neurol. $11: 451-463$.

23. Reivich, M., G. IsaAcs, E. Evarts, and S. Kety. 1968. The effect of slow wave sleep and REM sleep on regional cerebral blood flow in cats. J. Ncurochem. 15 : 301-306.

24. Smith, J. R., A. H. Nevis, and G. H. Collins. 1967. Polarization impedance of stainless steel bipolar electrodes in brain. Exp. Neurol. 18: 287-299.

25. TARBy, T. J., A. Costrn, and W. R. Adey. 1968. Effects of tetrodotoxin on impedance in normal and asphyxiated cerebral tissue. Exp. Neurol. 22 : 517-531.

26. Valverde, F. 1962. Reticular formation of the albino rat's brain stem sytoarchitecture and corticofugal connections. J. Comp. Neurol. 119: 25-49.

27. Van Harreveld, ., J. Crowell, and S. K. Malhotra. 1965. A study of extracellular spaces in central nervous tissue by freeze-substitution. $J$. C $\mathrm{cll} \mathrm{Biol}$. $25: 117-137$.

28. Van Harreveld, A., and F. I. Khattar. 1967. Changes in cortical extracellular space during spreading depression investigated with the electron microscope. J. Neurophysiol. 30 : 911-929.

29. Van Harreveld, A., and J. P. Schade. 1962. Changes in the electrical conductivity of cerebral cortex during seizure activity. Exp. Neurol. 5: 383-400.

30. WALker, F. D., and W. J. HiLD. 1969. Neuroglia electrically coupled to neurons. Science 165 : $602-603$.

31. WANG, H. H., and W. R. AnEy, 1969. Effects of cations and hyaluronidase on cerebral electrical impedance. Exp. Ncurol. 25 : 70-84. 